\title{
Protective effect of luteolin on cigarette smoke extract-induced cellular toxicity and apoptosis in normal human bronchial epithelial cells via the Nrf2 pathway
}

\author{
XIAOBIN TAN ${ }^{1 *}$, PING JIN ${ }^{1,2^{*}}$, LIANG FENG ${ }^{1}$, JIE SONG $^{1}$, E. SUN ${ }^{1}$, \\ WENBO LIU ${ }^{1}$, LUAN SHU ${ }^{1}$ and XIAOBIN JIA ${ }^{1}$ \\ ${ }^{1}$ Key Laboratory of New Drug Delivery System of Chinese Materia Medica, Jiangsu Provincial Academy of Chinese Medicine, \\ Nanjing, Jiangsu 210028; ${ }^{2}$ Department of Pharmaceutics, Huaihua Medical College, Huaihua, Hunan 418000, P.R. China
}

Received September 29, 2013; Accepted November 20, 2013

DOI: $10.3892 /$ or.2014.3007

\begin{abstract}
Luteolin, one of the most common abundant flavonoids in vegetables and herbs, has antitumor effects on various tumors by inducing apoptosis, antioxidant effects and inhibition of angiogenesis. However, the potential chemoprevention of luteolin on lung cell damage and its related mechanism(s) are not fully known. The present study evaluated the protective effects of luteolin on cigarette smoke extract (CSE)-induced toxicity and apoptosis in normal human bronchial epithelial (NHBE) cells and explored its underlying mechanism(s). MTT assay showed that pretreatment with luteolin increased CSE-decreased cell viability $(\mathrm{p}<0.05)$. Luteolin increased cellular glutathione (GSH) levels but decreased reactive oxygen species (ROS) generation $(\mathrm{p}<0.05)$. Cytometry assay and western blot analysis showed that luteolin attenuated CSE-induced apoptosis and apoptosis-related protein activation, including caspase $-3,-8$ and $-9(\mathrm{p}<0.05)$. The expression of CSE-induced NAD(P)H: quinone oxidoreductase 1 (NQO1) and heme oxygenase-1 (HO-1) were decreased significantly by luteolin $(\mathrm{p}<0.05)$. Furthermore, luteolin attenuated CSE-induced apoptosis, noticeably reduced CSE-induced expression of NF-E2-related factor 2 (Nrf2), NQO1 and HO-1 using a small interfering RNA (siRNA) transfection assay. The data demonstrated that CSE-induced oxidative damage and apoptosis through the Nrf2 pathway was inhibited by luteolin and it may serve as a chemopreventive agent for the prevention and treatment of lung cancer.
\end{abstract}

Correspondence to: Professor Xiaobin Jia, Key Laboratory of New Drug Delivery System of Chinese Materia Medica, Jiangsu Provincial Academy of Chinese Medicine, 100 Shizi Road, Nanjing 210028, P.R. China

E-mail: xiaobinjia_nj@126.com

${ }^{*}$ Contributed equally

Key words: luteolin, lung cancer, chemoprevention, cigarette smoking, apoptosis, NF-E2-related factor 2

\section{Introduction}

Lung cancer is one of the most commonly diagnosed types of cancer and the leading cause of cancer-related morbidity and mortality in the world (1). It is estimated that there will be approximately 228,000 new cases of lung cancer and 159,500 deaths in the United States in 2013 (2). Smoking, particularly smoking cigarette, is the most significant risk factor resulting in lung cancer (1). Cigarette smoke contains several types of chemical constituents, including tar, nicotine, polycyclic aromatic hydrocarbons, quinines and carbon monoxide. The lungs are the most important target organs for cigarette smoke-induced diseases. It has been shown that cigarette smoke extract (CSE) may produce a clear cytotoxic effect. It is well established that tentatively assigned to semiquinones present in aqueous extracts of CS are cytotoxic and cause protein and DNA damage and, therefore, cause lung cell damage or apoptosis (3-6). The smoking-induced pathological damage of epithelial cells was associated with the oxidative damage and transcription factor NF-E2-related factor 2 (Nrf2) (7,8). Activated-Nrf2 regulated the activity and expression of multiple genes and topoisomerase, including encoding xenobiotic metabolizing enzymes $\mathrm{NAD}(\mathrm{P}) \mathrm{H}$ : quinone oxidoreductase 1 (NQO1), heme oxygenase-1 (HO-1), superoxide dismutase (SOD), glutathione S-transferase (GST) and glutathione (GSH) (9-11). Therefore, preventing CSE-induced damage may be an effective method to reduce the high morbidity and mortality of lung cancer.

Luteolin, shown in Fig. 1, is one of the most common abundant flavonoids in vegetables and herbs, and has antitumor effects on various tumors by inducing apoptosis, antioxidant effects and anti-angiogenesis (12-17). Notably, in non-small cell lung cancer A549 cells, which possess constitutively active Nrf2, luteolin elicited a marked reduction in $\mathrm{Nrf} 2$ at both the mRNA and the protein levels, leading to decreasing Nrf2 binding to AREs, downregulation of ARE-driven genes, and depletion of reduced GSH (18).

It has been shown that luteolin has potent curative properties against $\mathrm{N}$-nitrosodiethylamine-induced hepatocellular carcinoma in albino rats and has potentiality in chemoprevention (19). However, the potential protection of luteolin 
on CSE-induced cell damage and apoptosis and its related mechanism has not yet been reported. In the present study, a normal human bronchial epithelial cell (NHBE) model was used to observe the influence of CSE on cell survival, apoptosis, DNA damage and intracellular stress response and luteolin intervention in CSE-induced cytotoxicity. Our findings showed that luteolin may be a promising chemopreventive agent for smoking-induced lung cancer.

\section{Materials and methods}

Reagents. RPMI-1640 medium was purchased from KeyGen (Nanjing, China). Luteolin was provided by Chengdu Must Biotechnology Co., Ltd. (Chengdu, China). Fetal bovine serum (FBS) was purchased from Gibco (USA). Nrf2, HO-1 and NQO1 antibodies were obtained from Santa Cruz Biotechnology, Inc. (USA). Cleaved caspase-3, -8 and -9, Bcl-2 and Bax antibodies were purchased from ImmunoWay Biotechnology Company (USA). The other chemicals and reagents used were of analytical grade.

Cell culture. NHBE cells were purchased from American Type Culture Collection (ATCC, Manassas, VA, USA). The NHBE cells were cultured with RPMI-1640 medium containing $10 \%$ FBS, penicillin $(80 \mathrm{U} / \mathrm{ml})$ and streptomycin $(0.08 \mathrm{mg} / \mathrm{ml})$. Cells were incubated at $37^{\circ} \mathrm{C}$ in a humidified atmosphere of 95\% air and $5 \% \mathrm{CO}_{2}$. The medium was replaced every day. For CSE treatment, NHBE cells were grown to $90 \%$ confluence and restored in fresh medium without FBS.

Preparation of CSE. CSE was prepared according to a method previously described $(20,21)$. In order to obtain $100 \%$ CSE, the filters were cut from the cigarettes before the experiment. One commercial cigarette (Nanjing, China) containing $11 \mathrm{mg}$ tar and $1.1 \mathrm{mg}$ nicotine was burning and drawing the smoke by vacuum into $10 \mathrm{ml}$ of cell growth medium at a rate of $5 \mathrm{~min}$. CSE was adjusted to $\mathrm{pH} 7.4$, filtered through a $0.22-\mu \mathrm{m}$ pore acaroids syringe filter (Pall, USA) and subsequently adjusted with medium to an absorbance of 0.15 at $320 \mathrm{~nm}$. The prepared medium containing CSE was used within 30 min of preparation.

MTT assay for cell viability. The effect of luteolin on NHBE cell viability was detected by the MTT assay. Cell suspension $\left(1 \times 10^{4}\right.$ cells $\left./ \mathrm{ml}\right)$ was seeded into wells of 96 -well plates. After incubation for $24 \mathrm{~h}$, the NHBE cells were pretreated with luteolin $(0.3125,0.625,1.25,2.5,5,10,20$ and $40 \mu \mathrm{M})$ and then treated with $10 \%$ CSE for another $24 \mathrm{~h}, 10 \%$ CSE was used to induce the NHBE damage model, while medium served as the negative control. At the end of the incubation, these cells were incubated with $0.5 \mathrm{mg} / \mathrm{ml}$ MTT for $4 \mathrm{~h}$. The purple crystals were dissolved by $100 \mu \mathrm{l}$ DMSO and were then shaken in a shaker for $10 \mathrm{~min}$. Finally, the absorbance of samples was measured in a microplate reader at 570 and $490 \mathrm{~nm}$. All the experiments were repeated 3 times.

$$
\text { Cell viability ratio }=\frac{A_{570 \text { treated }}-A_{490 \text { oreated }}}{A_{570 \text { control }}-A_{490 \text { control }}} \times 100 \%
$$

Reactive oxygen species (ROS) assay. The pre-fluorescent probe DCFH-DA was used to determine the intracellular<smiles>O=c1cc(-c2ccc(O)c(O)c2)oc2cc(O)cc(O)c12</smiles>

Figure 1. The chemical structure of luteolin.

ROS overgeneration induced by CSE according to the manufacturer's protocols (KeyGen). Briefly, after treatment with luteolin or (luteolin $+10 \%$ CSE), the cells were incubated with DCFH-DA $(10 \mu \mathrm{mol} / 1)$ for $20 \mathrm{~min}$ in an incubator in the dark at $37^{\circ} \mathrm{C}$. The fluorescence intensity was measured by a FACScan flow cytometer (BD, USA) at an excitation maximum of $488 \mathrm{~nm}$ and an emission maximum of $525 \mathrm{~nm}$.

Determination of GSH. Cells were collected from 6-well plates, washed with $4^{\circ} \mathrm{C}$ PBS twice and lysed in $200 \mu 1$ cell lysis buffer (Biyuntian Biotech. Co. Ltd., Nantong, China) with PMSF at $4^{\circ} \mathrm{C}$ for $30 \mathrm{~min}$, then centrifuged for $5 \mathrm{~min}\left(4^{\circ} \mathrm{C}, 13,000 \mathrm{rpm}\right)$, and the supernatant was used to detect cellular GSH level according to the manufacturer's protocols (NJJC-BIO, Nanjing, China). The final absorbance was detected at $405 \mathrm{~nm}$ in a microplate reader.

Apoptosis analysis by flow cytometry. NHBE cells $\left(1 \times 10^{6}\right.$ cells $\left./ \mathrm{ml}\right)$ were incubated with luteolin $+10 \%$ CSE for $24 \mathrm{~h}$. Then, the washed cells for apoptosis analysis were resuspended in Annexin V binding buffer and stained simultaneously with FITC-conjugated Annexin V-APC and PI at room temperature for $15 \mathrm{~min}$ in the dark, prior to the addition of binding buffer. The apoptotic cells were measured using a FACScan flow cytometer at an excitation maximum of $650 \mathrm{~nm}$ and an emission maximum of $660 \mathrm{~nm}$.

Western blot analysis. Cells were collected from 6-well plates and washed with PBS twice and then lysed in $200 \mu 1$ cell lysis buffer (Biyuntian Biotech. Co. Ltd.) with PMSF at $4^{\circ} \mathrm{C}$ for $30 \mathrm{~min}$. The cell lysis buffer was centrifuged for $5 \mathrm{~min}\left(4^{\circ} \mathrm{C}, 13,000 \mathrm{rpm}\right)$. The supernatant was separated by $12 \%$ of SDS-PAGE. The SDS-separated proteins were equilibrated in transfer buffer (50 mM Tris-HCl, pH 6.8, $40 \mathrm{mM}$ glycine, $10 \%$ SDS and 20\% methanol) and electrotransferred to Immobilon-P transfer membranes (Millipore Corporation, Bedford, MA, USA). The blot was blocked with 5\% non-fat dry milk and washed with Tris-buffered saline $10 \mathrm{mM}$ Tris, $150 \mathrm{mM} \mathrm{NaCl}$ (Sigma Chemical). Subsequently, membranes were probed with the primary antibodies against Bcl-2, BAX, cleaved caspase-3, -8 and -9 , Nrf2, NQO1, HO-1 and $\beta$-actin. Membranes were washed twice with TBST, the horseradish peroxidase-bound secondary antibodies were added to membranes for $1 \mathrm{~h}$ at room temperature. Membranes were visualized with chemiluminescence reagents. Image Pro Plus (IPP) software for densitometry analysis was applied for the quantification of protein expressions. 

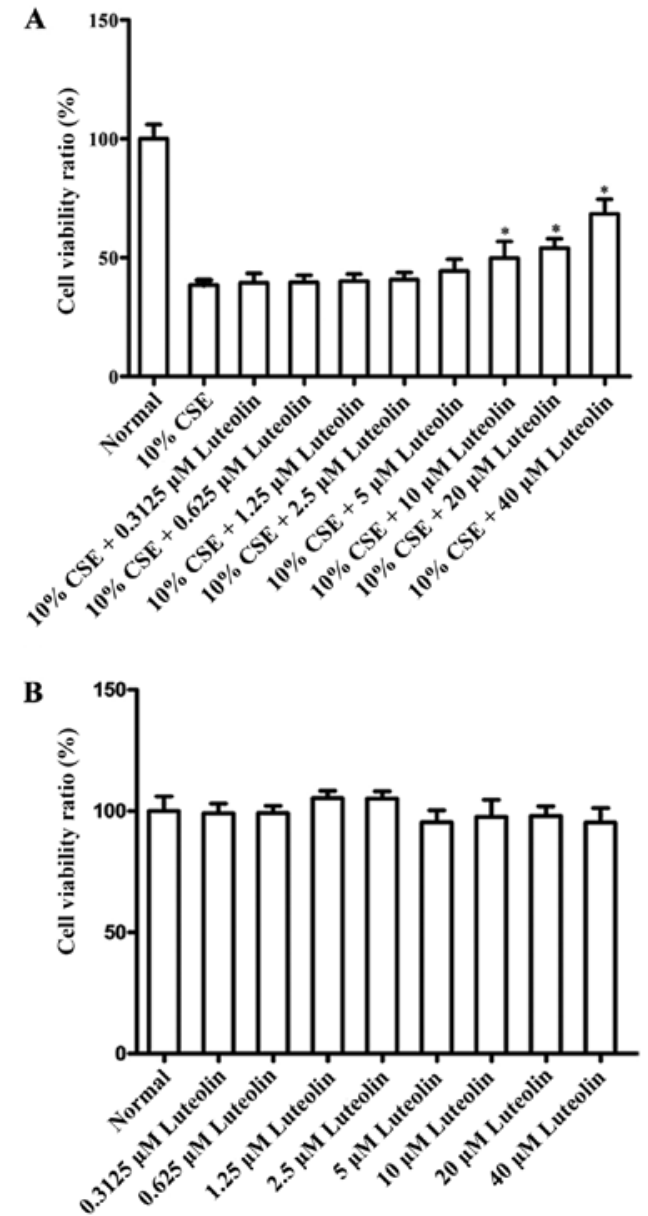

Figure 2. Effect of luteolin on the cell viability in NHBE cells. (A) Cells were treated with $10 \%$ CSE + luteolin for $24 \mathrm{~h}$. (B) Cells were treated with single luteolin for $24 \mathrm{~h}$. The cell viability was determined by MTT assay. Values are means \pm SD of 3 independent experiments. ${ }^{*} p<0.05$, (10\% CSE + luteolin) vs. CSE. NHBE, normal human bronchial epithelial; CSE, cigarette smoke extract.

Small interfering RNA (siRNA) transfection assay. For the transfections, NHBE cells were seeded $\left(1 \times 10^{5}\right.$ cells $\left./ \mathrm{ml}\right)$ in 6 -well plates with antibiotic-free normal growth medium supplemented with FBS. For each transfection, $0.8 \mathrm{ml}$ siRNA transfection medium (sc-36868; Santa Cruz Biotechnology, Inc.) were added to each tube containing the siRNA transfection reagent mixture (solution $\mathrm{A}+$ solution $\mathrm{B}$ ). The directed against human Nrf2 (sc-37049) and control-siRNA (a non-targeting siRNA; sc-37049) (both from Santa Cruz Biotechnology, Inc.) were transfected into NHBE cells using the Lipofectamine ${ }^{\mathrm{TM}} 2000$ reagent according to the manufacturer's instructions. NHBE cells were incubated with the transfection complexes for $24 \mathrm{~h}$ and subsequently analyzed by western blotting for protein expression. The effects of Nrf2 siRNA were compared with those of corresponding control siRNA using the same transfection method. This approach was used to verify the direct link between luteolin, Nrf2 and CSE.

Statistical analysis. The data from three individual experiments are presented as means \pm standard deviation (SD). Statistical analysis was performed by one-way analysis of variance (ANOVA) followed by Tukey's test using SPSS 16.0 software. $\mathrm{p}<0.05$ was considered to indicate a statistically significant difference.
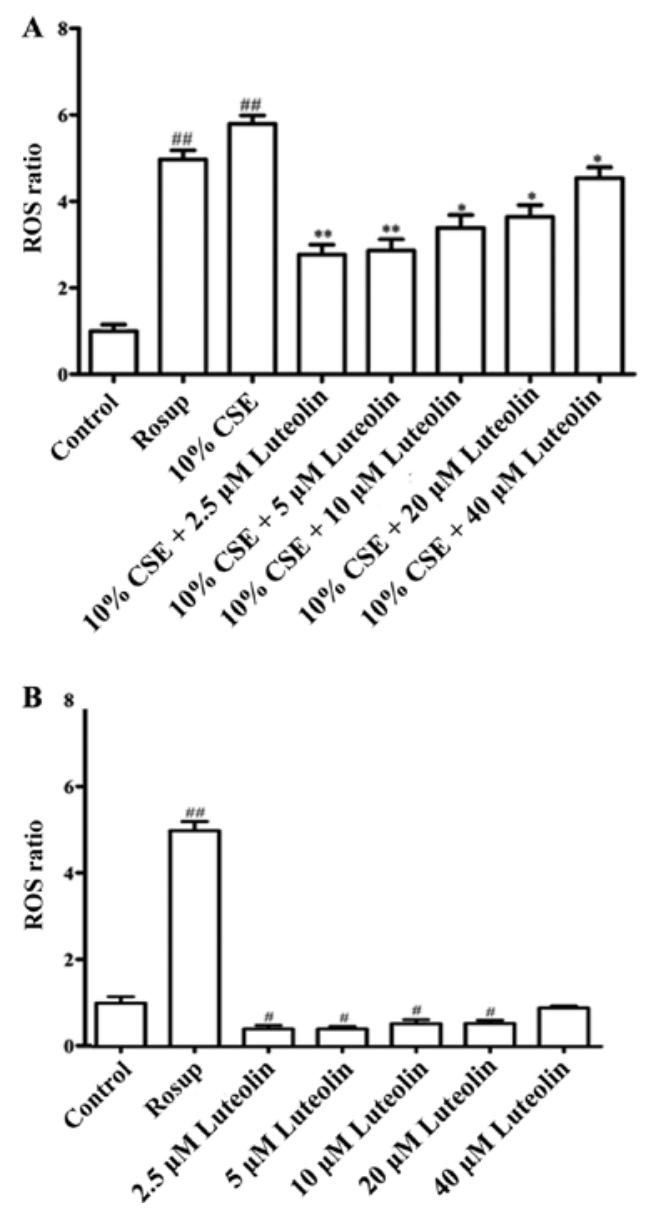

Figure 3. The reduction of luteolin on CSE-induced ROS overgeneration. (A) Intracellular ROS by $10 \%$ CSE + luteolin. (B) Intracellular ROS by luteolin; " $\mathrm{p}<0.05,{ }^{* *} \mathrm{p}<0.0110 \%$ CSE vs. $10 \%$ CSE + luteolin; ${ }^{\# p}<0.05,{ }^{\# \#} \mathrm{p}<0.01$ control vs. $10 \%$ CSE or luteolin. CSE, cigarette smoke extract; ROS, reactive oxygen species.

\section{Results}

Effect of luteolin on CSE-induced NHBE cell viability. Several studies reported that the toxicity induced by CSE may significantly inhibit the viability of NHBE cells in light of a potentially pathogenic event, such as oxidative stress $(4,22)$. Herein, we observed that the exposure to CSE may markedly reduce NHBE cell viability to $38.55 \pm 2.13 \%$ ( $<<0.05$, CSE vs. normal). Pretreatment with luteolin at the dose of $0.3125-40 \mu \mathrm{M}$ significantly improved the cell viability (Fig. 2A). However, the single luteolin at the same range of concentrations had no effect on the cell viability after being treated for $24 \mathrm{~h}$ (Fig. 2B). The data indicated that luteolin attenuated CSE-induced cellular damage and significantly improved the cell viability in NHBE cells.

Luteolin reduces CSE-induced ROS overgeneration. As shown in Fig. 3A, 10\% CSE triggered oxidative stress and significantly increased intracellular ROS overgeneration as compared to the normal control $(\mathrm{p}<0.05)$. Markedly, CSE-induced overgeneration of ROS was inhibited significantly by the treatment with single luteolin or luteolin $+10 \%$ CSE in a concentrationdependent manner (Fig. 3A and B). The results showed that luteolin reduced the oxidative damage in NHBE cells. 


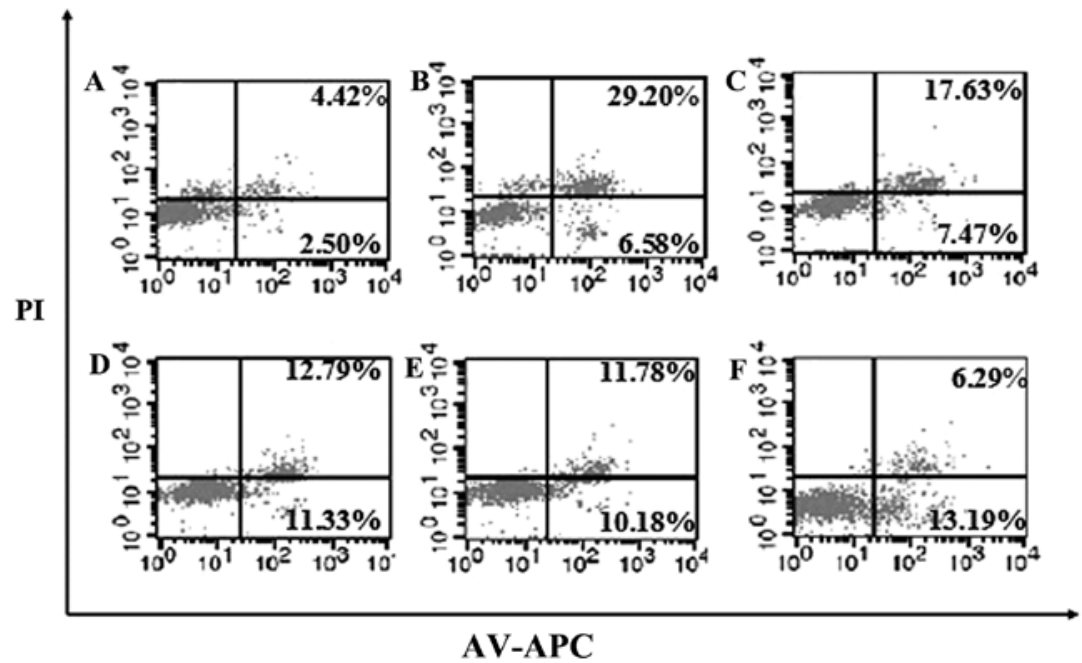

Figure 4. Luteolin prevents CSE-induced NHBE cell apoptosis. NHBE cells were pretreated with luteolin $+10 \% \mathrm{CSE}$ for $24 \mathrm{~h}$ and stained with Annexin V-PI kit. (A) Control, (B) 10\% CSE, (C) 10\% CSE $+5 \mu \mathrm{M}$ luteolin, (D) 10\% CSE $+10 \mu \mathrm{M}$ luteolin, (E) $10 \% \mathrm{CSE}+20 \mu \mathrm{M}$ luteolin, (F) $10 \% \mathrm{CSE}+40 \mu \mathrm{M}$ luteolin. CSE, cigarette smoke extract; NHBE, normal human bronchial epithelial.
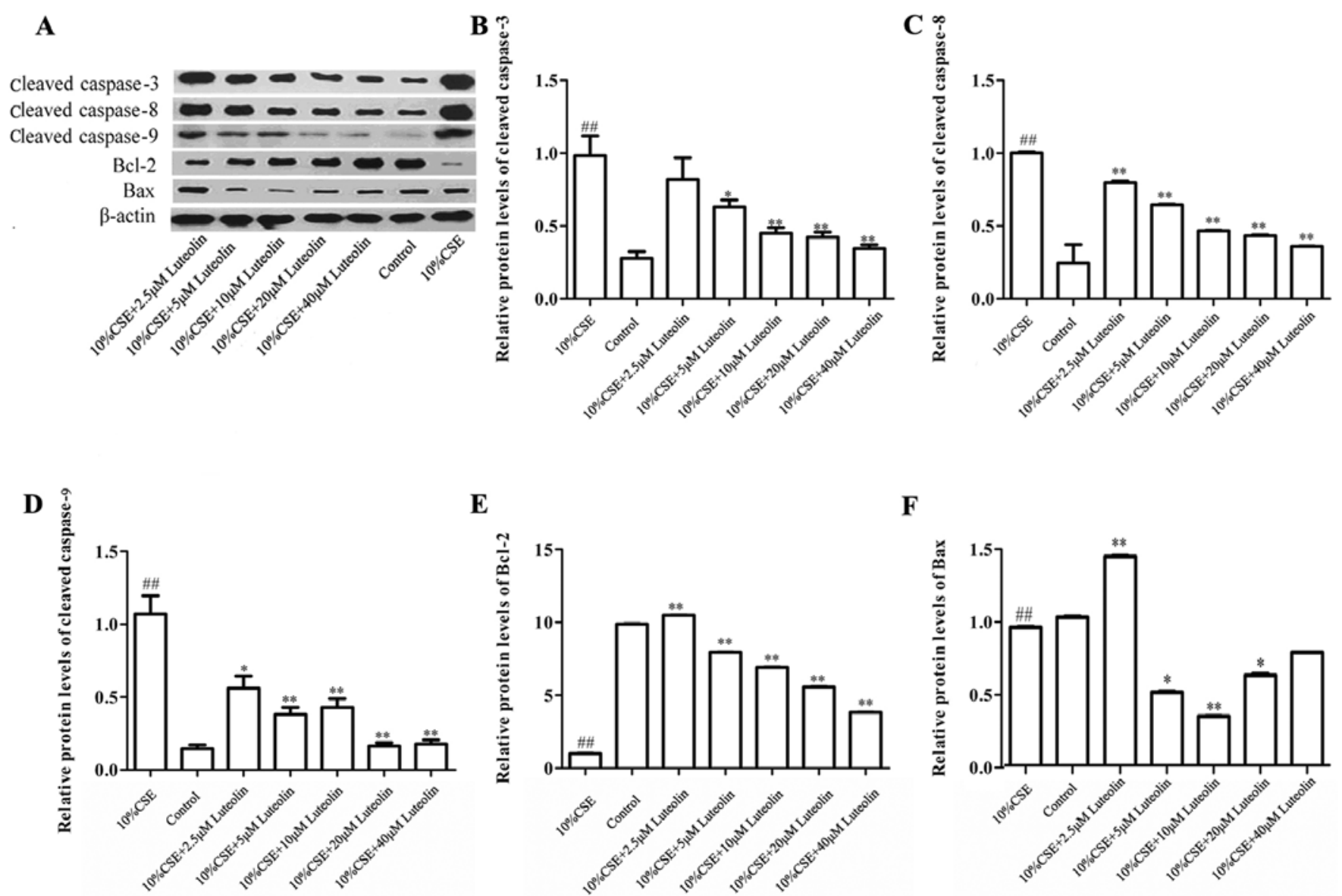

Figure 5. The effects of luteolin on CSE-induced expressions of apoptotic proteins. (A) NHBE cells were treated with $10 \% \mathrm{CSE}+$ luteolin for $24 \mathrm{~h}$ and cleaved caspase- $3,-8$ and -9 , Bax and Bcl-2 were analyzed by western blotting; (B) cleaved caspase- 3 expression; (C) cleaved caspase- 8 expression; (D) cleaved caspase-9 expression; (E) Bcl-2 expression; (F) Bax expression. ${ }^{* \#} \mathrm{p}<0.01$ control vs. CSE; ${ }^{*} \mathrm{p}<0.05,{ }^{* * *} \mathrm{p}<0.01 \mathrm{CSE}$ vs. $10 \%$ CSE + luteolin. CSE, cigarette smoke extract; NHBE, normal human bronchial epithelial.

Luteolin reduces CSE-induced NHBE cell apoptosis. To examine whether luteolin prevents CSE-induced NHBE cell

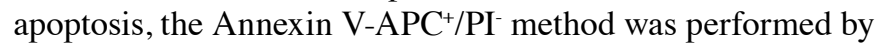

flow cytometry in the present study. As shown in Fig. 4, after being treated with $10 \%$ CSE, the apoptosis ratio of NHBE cells was $35.78 \%$. The statistical results showed that there 
A

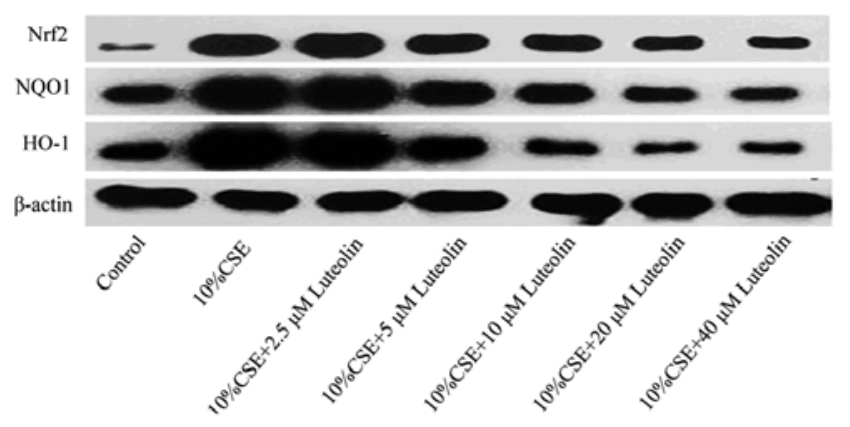

B

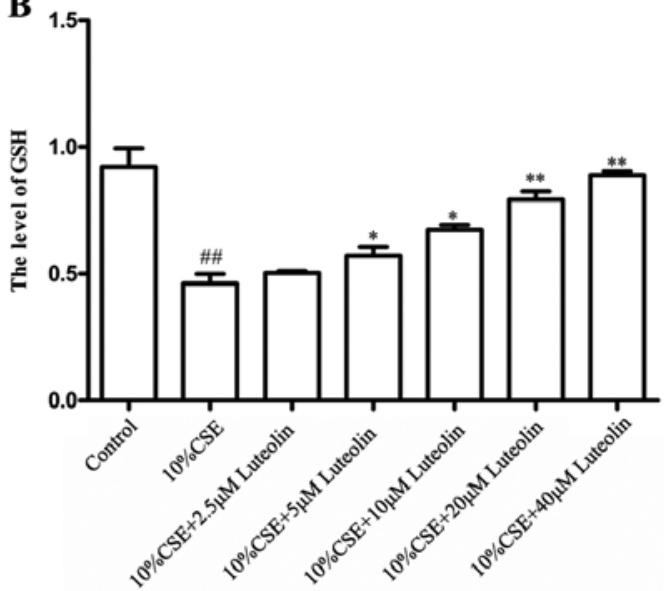

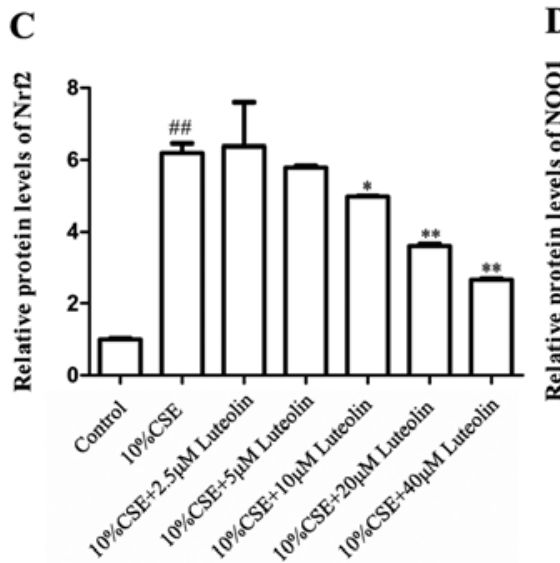

D

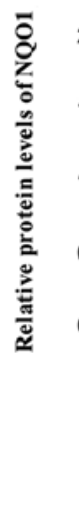

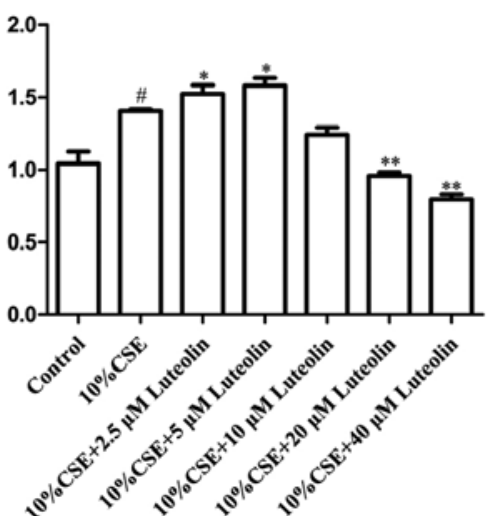

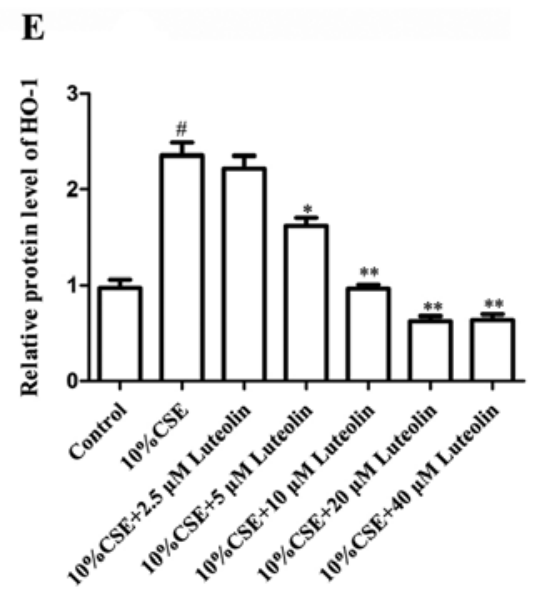

Figure 6. The effects of luteolin on the Nrf2 signaling pathway in NHBE cells. (A) Cells were treated with 10\% CSE + luteolin, and Nrf2, NQO1 and HO-1 expressions were reduced when compared with $10 \%$ CSE; (B) luteolin recovered intracellular GSH level; (C) the protein level of Nrf2 expression was semiquantified by densitometry analysis; (D) the protein level of NQO1 expression; (E) the protein level of HO-1 expression. ${ }^{\#}$ p $<0.05$, ${ }^{\# \#}$ p $<0.01$ control vs. CSE; ${ }^{*} \mathrm{p}<0.05,{ }^{* *} \mathrm{p}<0.01 \mathrm{CSE}$ vs. 10\% CSE + luteolin. Nrf2, NF-E2-related factor 2; NHBE, normal human bronchial epithelial; CSE, cigarette smoke extract; NQO1, $\mathrm{NAD}(\mathrm{P}) \mathrm{H}$ : quinone oxidoreductase 1 ; $\mathrm{HO}-1$, heme oxygenase-1; GSH, glutathione.

was a significant difference between $10 \%$ CSE groups and normal control $(6.92 \%)$. Notably, the apoptosis ratio of cells treated with luteolin $(5-40 \mu \mathrm{M})+10 \%$ CSE was lower than that of $10 \%$ CSE (35.78\%); the apoptosis ratios of NHBE cells were $19.48,21.96,24.02$ and $25.10 \%$, respectively. The results showed that luteolin prevented CSE-induced NHBE cell apoptosis in a concentration-dependent manner.

Luteolin downregulates CSE-induced activation of caspases and Bax/Bcl-2 expression. Caspase-3, -8 and -9, Bax and Bcl-2 were the major effector proteins and played a critical role in the characteristic apoptotic events, including chromatin condensation, DNA fragmentation and the formation of apoptotic bodies. Therefore, the effect of luteolin on CSE-induced activation of caspase- $3,-8$ and -9 was investigated. As shown in Fig. 5, CSE exposure markedly increased the activation of caspase-3, -8 and -9 , and upregulated the ratio of $\mathrm{Bax} / \mathrm{Bcl}-2$ in NHBE cells. These data strongly indicated that the protective effect of luteolin against CSE-induced apoptosis in NHBE cells was through modulating the activation of caspase-3, -8 and -9 and reducing the ratio of $\mathrm{Bax} / \mathrm{Bcl}-2$.
Luteolin attenuates CSE-induced Nrf2, NQO1 and HO-1 protein expressions and improves GSH level. In order to evaluate whether luteolin attenuates CSE-induced oxidative stress or electrophilic attack by activating Nrf2-mediated signaling pathway, Nrf2, NQO1 and HO-1 protein expressions were analyzed by western blot analysis while antioxidant GSH was detected by kits. Following treatment with $10 \%$ CSE in the presence of luteolin $(2.5,5,10,20$ and $40 \mu \mathrm{M})$ for $24 \mathrm{~h}$, the Nrf2, NQO1 and HO-1 protein expressions in NHBE cells were enhanced by the exposure of $10 \% \mathrm{CSE}$, when compared with the normal group $(\mathrm{p}<0.01)$ (Fig. 6A). The treatment with luteolin (2.5-40 $\mu \mathrm{M})$ may significantly attenuate the protein expressions of $\mathrm{Nrf} 2(\mathrm{p}<0.01)$. Additionally, the expressions of downstream signaling NQO1 and HO-1 proteins associated with $\mathrm{Nrf} 2$ activation were also markedly reduced by the treatment with luteolin $(\mathrm{p}<0.01)$. The results showed that the attenuation of luteolin on $10 \%$ CSE-induced oxidative damage may be associated with the blockade on Nrf2-mediated signaling pathway. GSH played an important role in the Nrf2mediated antioxidant defense system in organism. To examine the effect of luteolin on $10 \%$ CSE-induced oxidative damage, 
A
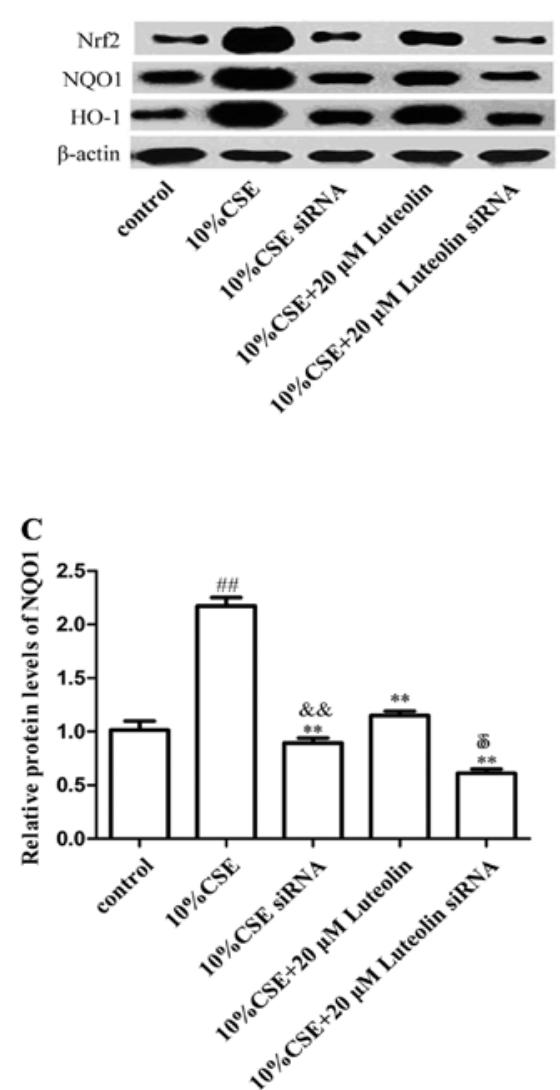
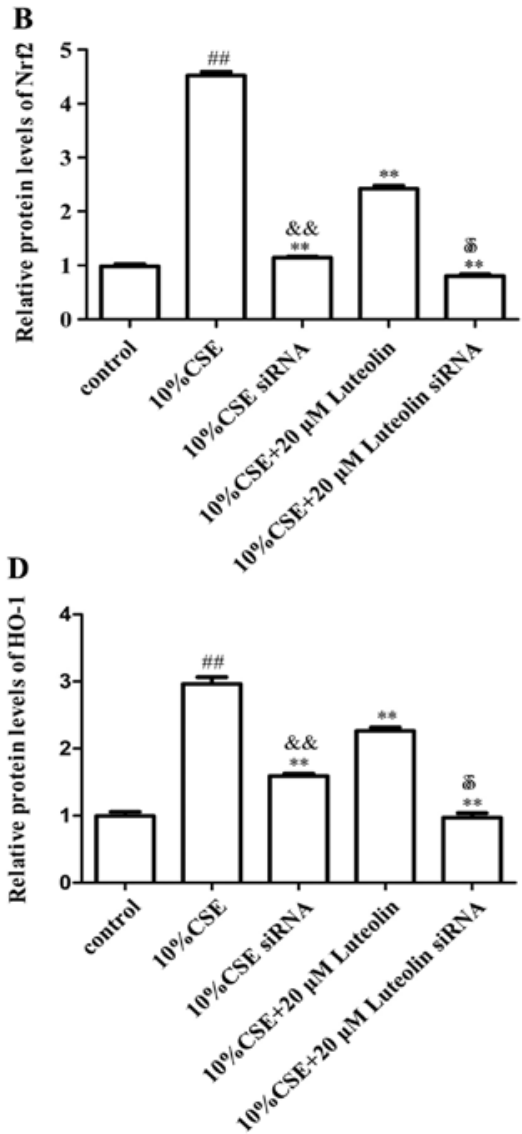

Figure 7. The Nrf2 pathway is specifically responsible for luteolin-induced cytoprotection. (A) Nrf2 siRNA is effective in silencing Nrf2 translocation and activation of target genes. After transfection, cells were exposed to 10\% CSE or 10\% CSE + luteolin for $24 \mathrm{~h}$, and the protein levels of Nrf2, NQO1 and HO-1 were confirmed by western blot analysis. (B-D) The protein levels of Nrf2, NQO1 and HO-1 expressions were semi-quantified by densitometry analysis.

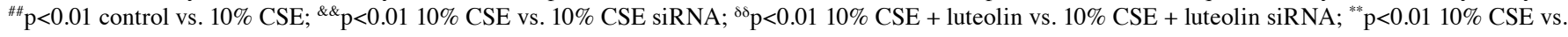
experimental group. Nrf2, NF-E2-related factor 2; siRNA, small interfering RNA; CSE, cigarette smoke extract; NQO1, NAD(P)H: quinone oxidoreductase 1; HO-1, heme oxygenase-1.

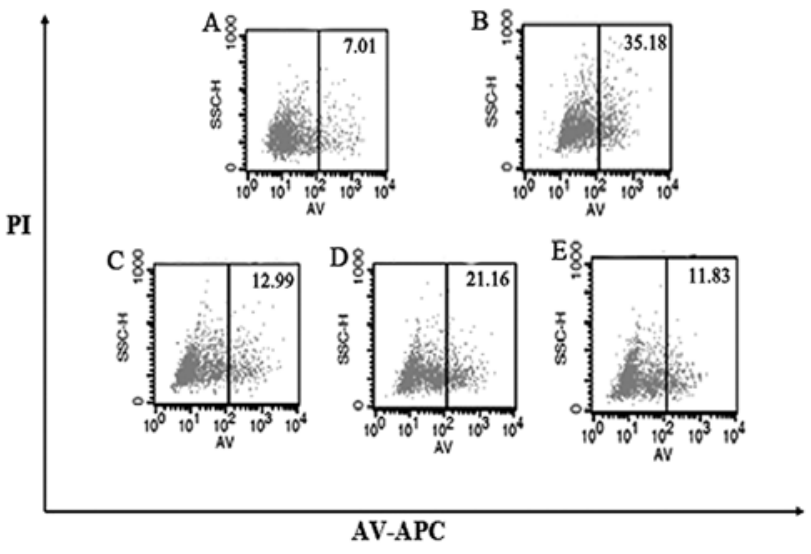

Figure 8 . The Nrf2 pathway specifically responsible for luteolin prevented CSE-induced NHBE cell apoptosis. After transfection, cells were exposed to $10 \% \mathrm{CSE}$ or $10 \% \mathrm{CSE}+$ luteolin for $24 \mathrm{~h}$ and stained with Annexin V-PI kit. (A) Control, (B) $10 \%$ CSE, (C) $10 \%$ CSE + siRNA, (D) $10 \%$ CSE $+20 \mu \mathrm{M}$ luteolin, and (E) $10 \% \mathrm{CSE}+20 \mu \mathrm{M}$ luteolin + siRNA. Nrf2, NF-E2-related factor 2; CSE, cigarette smoke extract; NHBE, normal human bronchial epithelial; siRNA, small interfering RNA.

the level of intracellular GSH in NHBE cells was measured. Luteolin $(2.5-40 \mu \mathrm{M})$ significantly reduced $10 \%$ CSE-induced intracellular GSH level from 0.461 to 0.889 ( $\mathrm{p}<0.01,10 \%$ CSE vs. luteolin $+10 \%$ CSE) (Fig. 6B). The results indicated that the reduction of luteolin on 10\% CSE-induced GSH level may contribute to Nrf2-mediated oxidative damage.

Nrf2 knockdown diminishes the protective effect of luteolin in NHBE cells. To evaluate the role of the Nrf2 pathway in the protection of luteolin against CSE-induced toxicity, we developed an Nrf2 gene knockdown model in NHBE cells by using siRNA transfection. After transfection for $48 \mathrm{~h}$, cells were treated with $10 \% \mathrm{CSE}$ or $10 \% \mathrm{CSE}+20 \mu \mathrm{M}$ luteolin for $4 \mathrm{~h}$. Gene silencing against Nrf2 suppressed CSE-induced translocation of Nrf2 and the expression of NQO1, HO-1 and apoptosis in NHBE cells (Figs. 7 and 8). The protein levels of Nrf2, NQO1 and HO-1 were confirmed by western blot anal-

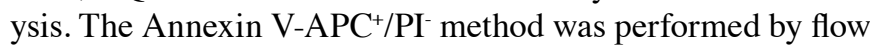
cytometry in the present study. The transfection of siNrf2 led to the reduction in the Nrf2, NQO1 and HO-1 protein level and decreased the CSE-induced Nrf2 expression in NHBE cells $(\mathrm{p}<0.05)$. A significant difference was observed between the $10 \%$ CSE groups (35.18\%) and 10\% CSE + siRNA (12.99\%). Of note, the apoptosis ratio of cells treated with $20 \mu \mathrm{M}$ luteolin $+10 \%$ CSE + siRNA (11.83\%) was lower than that of $10 \% \mathrm{CSE}+20 \mu \mathrm{M}$ luteolin $(21.16 \%)$. These results indicated that the Nrf2 pathway played a key role in the luteolin-induced cytoprotection against CSE. 


\section{Discussion}

Cigarette smoke extract (CSE), a mixture of $>4,000$ chemicals, includes significant amounts of free radicals, particles, reactive chemicals and gases, producing an overwhelming oxidative burden on the lungs $(23,24)$. Cigarette smoking is considered a major cause of morbidity and mortality of lung cancer $(25,26)$. In China, there is a large number of smoking and passive smoking populations (27). The CSE-induced lung injuries on those people are mainly correlated with oxidative stress damage (28). The long-lived radicals presented in aqueous extracts of CSE were cytotoxic and caused protein and DNA damage resulting in the occurrence and development of lung cancer $(29,30)$. Additionally, accumulating evidence has indicated that CSE may produce a clear cytotoxic effect on normal human bronchial epithelial (NHBE) cells (4,6,31-34). In our present study, the NHBE cell model was used to explore the protection of luteolin against the CSE-induced damage.

Chemoprevention, an effective prevention and treatment measure for lung and other types of cancer, has been defined as using natural, synthetic substances to prevent, inhibit or reverse the initiation and development of precancerous lesions through reducing the morbidity and mortality of cancer. Recent studies showed that chemoprevention is considered a potential approach to control lung cancer (35). In the present study, the chemoprevention of luteolin on CSE-induced NHBE cell damage was performed to explore the potential benefits and underlying mechanism.

The inhibition on apoptosis of normal cells plays a crucial role in the prevention process of lung cancer. Apoptosis, a highly controlled physiological process and a core signaling pathway, may be triggered by stimuli, such as CSE (36). In the present study, we detected the protein of Bax/Bcl-2 and caspase series caspase- $3,-8$ and -9 related mitochondrial pathways by western blot analysis. Our data indicated that luteolin downregulated CSE-induced activation of caspases and Bax/ Bcl-2 expression.

Oxidative stress is caused by an imbalance of reactive oxygen species (ROS)/reactive nitrogen species (RNS) and the antioxidative stress defense systems in cells. ROS/RNS or carcinogen metabolites may attack intracellular proteins, lipids and nucleic acids, which may result in genetic mutations, carcinogenesis and other diseases. In the present study, 10\% CSE was used to induce oxidative stress in NHBE cells. Notably, luteolin may attenuate 10\% CSE-induced oxidative stress. In addition, we observed an intracellular GSH level increased by the treatment with luteolin. Since $\gamma$-glutamylcysteine synthetase, a phase II enzyme regulated by the Nrf2 pathway, is the rate-limiting enzyme in GSH biosynthesis, the GSH level increase may reflect the induction of $\gamma$-glutamylcysteine synthetase by luteolin $(37,38)$.

Nrf2, a redox-sensitive transcription factor, has emerged as a novel target for chemoprevention. Experimental evidence shows that Nrf2 protects cells from oxidative stresses by activating the expression of a battery of cytoprotective genes via the antioxidant response element (39). Nrf2 plays a critical role in the regulation of many antioxidative stress/antioxidant and detoxification enzyme genes, such as xenobiotic metabolizing enzymes [NAD(P)H: quinone oxidoreductase 1 (NQO1), heme oxygenase-1 (HO-1), superoxide dismutase (SOD), glutathione
S-transferase (GST), glutathione (GSH)] (9), directly via the antioxidant response element. Several studies have shown that dietary phytochemicals possess cancer chemopreventive potential through the induction of Nrf2-mediated antioxidant/ detoxification enzymes to protect organisms against cellular damage caused by oxidative stress (40). The present study showed that luteolin protects cells from CSE-induced toxicity through activation of the Nrf2 pathway. Here, we confirmed that luteolin induced Nrf2 translocation to the nucleus and activated its downstream target genes encoding proteins in NHBE cells, such as phase II enzymes NQO1 and HO-1. Induction of NQO1 and HO-1 has been established as strong cellular defense against oxidative stress. This is consistent with the previous reports on the protection of the Nrf2 signaling pathway against chemical carcinogen-induced cytotoxicity (41-43).

Luteolin-induced antioxidative activities were deleted as Nrf2 was knocked down, indicating a specific role of the Nrf2 pathway in luteolin-induced cytoprotection against CSE. The discovery of the target pathway was significant as it provided a signal, which leads to identifying compounds with better efficacy. In the present study, we found that the luteolin-induced cytoprotection showed a clear dose-dependent manner $(0-40 \mu \mathrm{M})$. Therefore, luteolin may have optimal Nrf2-mediated antioxidative protection effect. However, further preclinical and clinical studies on the protective activity of luteolin on CSE toxicity need to be performed.

In conclusion, in the present study, we found that luteolin has a cytoprotective effect on CSE-induced NHBE cells and it may protect cells from CSE-induced toxicity through the activation of the Nrf2 pathway. Luteolin may act as a chemopreventive agent for the prevention and treatment of lung cancer.

\section{Acknowledgements}

The present study was supported by the Pharmaceutical Science and the Technology Project of Jiangsu Province (LZ11062).

\section{References}

1. Jemal A, Bray F, Center MM, et al: Global cancer statistics. CA Cancer J Clin 61: 69-90, 2011.

2. Siegel R, Naishadham D and Jemal A: Cancer statistics 2013. CA Cancer J Clin 63: 11-30, 2013.

3. Nakayama T, Kaneko M, Kodama M and Nagata C: Cigarette smoke induces DNA single-strand breaks in human cells. Nature 314: 462-464, 1985.

4. Nyunoya T, Monick MM, Klingelhutz A, et al: Cigarette smoke induces cellular senescence. Am J Respir Cell Mol Biol 35: 681-688, 2006

5. Carnevali S, Petruzzelli S, Longoni B, et al: Cigarette smoke extract induces oxidative stress and apoptosis in human lung fibroblasts. Am J Physiol Lung Cell Mol Physiol 284: L955-L963, 2003.

6. Baglole CJ, Bushinsky SM, Garcia TM, et al: Differential induction of apoptosis by cigarette smoke extract in primary human lung fibroblast strains: implications for emphysema. Am J Physiol Lung Cell Mol Physiol 291: L19-L29, 2006.

7. Kensler TW, Wakabayashi $\mathrm{N}$ and Biswal S: Cell survival responses to environmental stresses via the Keap1-Nrf2-ARE pathway. Annu Rev Pharmacol Toxicol 47: 89-116, 2007.

8. So HS, Kim HJ, Lee JH, et al: Flunarizine induces Nrf2-mediated transcriptional activation of heme oxygenase-1 in protection of auditory cells from cisplatin. Cell Death Differ 13: 1763-1775, 2006.

9. Leiser SF and Miller RA: Nrf2 signaling a mechanism for cellular stress resistance in long-lived mice. Mol Cell Biol 30: 871-884, 2010. 
10. Thimmulappa RK, Mai KH, Srisuma S, et al: Identification of Nrf2-regulated genes induced by the chemopreventive agent sulforaphane by oligonucleotide microarray. Cancer Res 62: 5196-5203, 2002.

11. Hayashi A, Suzuki H, Itoh K, et al: Transcription factor Nrf2 is required for the constitutive and inducible expression of multidrug resistance-associated protein 1 in mouse embryo fibroblasts. Biochem Biophys Res Commun 310: 824-829, 2003.

12. Ko WG, Kang TH, Lee SJ, et al: Effects of luteolin on the inhibition of proliferation and induction of apoptosis in human myeloid leukaemia cells. Phytother Res 16: 295-298, 2002.

13. Lee HZ, Wu CH and Chang SP: Release of nucleophosmin from the nucleus: involvement in aloe-emodin-induced human lung non small carcinoma cell apoptosis. Int J Cancer 113: 971-976, 2005.

14. Huang YT, Hwang JJ, Lee PP, et al: Effects of luteolin and quercetin, inhibitors of tyrosine kinase, on cell growth and metastasis-associated properties in A43 cells over expressing epidermal growth factor receptor. Br J Pharmacol 128: 999-1010, 1999.

15. Leung $\mathrm{HW}, \mathrm{Wu} \mathrm{CH}, \mathrm{Lin} \mathrm{CH}$ and Lee HZ: Luteolin induced DNA damage leading to human lung squamous carcinoma $\mathrm{CH} 27$ cell apoptosis. Eur J Pharmacol 508: 77-83, 2005.

16. Bagli E, Stefaniotou M, Morbidelli L, et al: Luteolin inhibits vascular endothelial growth factor-induced angiogenesis; inhibition of endothelial cell survival and proliferation by targeting phosphatidylinositol 3'-kinase activity. Cancer Res 64: 7936-7946, 2004.

17. Lin Y, Shi R, Wang X and Shen HM: Luteolin, a flavonoid with potential for cancer prevention and therapy. Curr Cancer Drug Targets 8: 634-646, 2008

18. Tang X, Wang H, Fan L, et al: Luteolin inhibits Nrf2 leading to negative regulation of the Nrf2/ARE pathway and sensitization of human lung carcinoma A549 cells to therapeutic drugs. Free Radic Biol Med 50: 1599-1609, 2011.

19. Balamurugan $\mathrm{K}$ and Karthikeyan J: Evaluation of luteolin in the prevention of N-nitrosodiethylamine-induced hepatocellular carcinoma using animal model system. Indian J Clin Biochem 27: 157-163, 2012.

20. Smelter DF, Sathish V, Thompson MA, et al: Thymic stromal lymphopoietin in cigarette smoke-exposed human airway smooth muscle. J Immunol 185: 3035-3040, 2010.

21. Hudy MH, Traves SL, Wiehler S and Proud D: Cigarette smoke modulates rhinovirus induced airway epithelial chemokine production. Eur Respir J 35: 1256-1263, 2010.

22. Albino AP, Huang X, Jorgensen ED, et al: Induction of DNA double-strand breaks in A549 and normal human pulmonary epithelial cells by cigarette smoke is mediated by free radicals. Int J Oncol 28: 1491-1505, 2006.

23. Cheng SE, Lee IT, Lin CC, et al: Cigarette smoke particle-phase extract induces HO-1 expression in human tracheal smooth muscle cells: role of the c-Src/NADPH oxidase/MAPK/Nrf2 signaling pathway. Free Radic Biol Med 48: 1410-1422, 2010.

24. Pryor WA and Stone K: Oxidants in cigarette smoke. Radicals, hydrogen peroxide, peroxynitrate, and peroxynitrite. Ann NY Acad Sci 686: 12-27, 1993.

25. Newcomb PA and Carbone PP: The health consequences of smoking. Cancer Med Clin North Am 76: 305-311, 1992.

26. Thun MJ, Henley SJ and Calle EE: Tobacco use and cancer: an epidemiologic perspective for geneticists. Oncogene 21: 7307-7325, 2002.
27. Poulsen HE, Prieme $\mathrm{H}$ and Loft S: Role of oxidative DNA damage in cancer initiation and promotion. Eur J Cancer Prev 7: 9-16, 1998.

28. Prasad KN and Edwards-Prasad J: Vitamin E and cancer prevention: recent advances and future potentials. J Am Coll Nutr 11: 487-500, 1992.

29. Banerjee S, Chattopadhyay R, Ghosh A, et al: Cellular and molecular mechanisms of cigarette smoke-induced lung damage and prevention by vitamin C. J Inflamm 11: 5-21, 2008.

30. Lannan S, Donaldson K, Brown D, et al: Effects of cigarette smoke and its condensates on alveolar epithelial cell injury in vitro. Am J Physiol 266: 92-100, 1994.

31. Nakamura Y, Romberger DJ, Tate L, et al: Cigarette smoke inhibits lung fibroblast proliferation and chemotaxis. Am J Respir Crit Care Med 151: 1497-1503, 1995.

32. van der Vaart H, Postma DS, Timens W and ten Hacken NH: Acute effects of cigarette smoke on inflammation and oxidative stress: a review. Thorax 59: 713-721, 2004.

33. Deslee G, Adair-Kirk TL, Betsuyaku T, et al: Cigarette smoke induces nucleic-acid oxidation in lung fibroblasts. Am J Respir Cell Mol Biol 43: 576-584, 2010.

34. Messner B, Frotschnig S, Steinacher-Nigisch A, et al: Apoptosis and necrosis: two different outcomes of cigarette smoke condensate-induced endothelial cell death. Cell Death Dis 3: e424, 2012.

35. Pan J, Zhang Q, Wang Y and You M: 226S proteasome activity is down-regulated in lung cancer stem-like cells propagated in vitro. PLoS One 5: e13298, 2010.

36. Comer DM, Kidney JC, Ennis M and Elborn JS: Airway epithelial cell apoptosis and inflammation in COPD, smokers and nonsmokers. Eur Respir J 41: 1058-1067, 2013.

37. Pandurangan AK, Ananda Sadagopan SK, Dharmalingam $P$ and Ganapasam S: Luteolin, a bioflavonoid inhibits Azoxymethaneinduced colorectal cancer through activation of Nrf2 signaling. Toxicol Mech Methods: Oct 7, 2013 (Epub ahead of print).

38. Huang CS, Lii CK, Lin AH, et al: Protection by chrysin, apigenin, and luteolin against oxidative stress is mediated by the Nrf2dependent up-regulation of heme oxygenase 1 and glutamate cysteine ligase in rat primary hepatocytes. Arch Toxicol 87: $167-178,2013$.

39. Florczyk U, Łoboda A, Stachurska A, et al: Role of Nrf2 transcription factor in cellular response to oxidative stress. Postepy Biochem 56: 147-155, 2010 (In Polish).

40. Su ZY, Shu L, Khor TO, et al: A perspective on dietary phytochemicals and cancer chemoprevention: oxidative stress, nrf2, and epigenomics. Top Curr Chem 329: 133-162, 2013.

41. Shinkai Y, Sumi D, Fukami I, et al: Sulforaphane, an activator of Nrf2, suppresses cellular accumulation of arsenic and its cytotoxicity in primary mouse hepatocytes. FEBS Lett 580: 1771-1774, 2006.

42. Osburn WO and Kensler TW: Nrf2 signaling: an adaptive response pathway for protection against environmental toxic insults. Mutat Res 659: 31-39, 2008.

43. Du Y, Villeneuve NF, Wang XJ, et al: Oridonin confers protection against arsenic-induced toxicity through activation of the Nrf2-mediated defensive response. Environ Health Perspect 116: 1154-1161, 2008 